\title{
Diagnosis of Gram negative, ventilator associated pneumonia by assaying endotoxin in bronchial lavage fluid
}

\author{
P G Flanagan, S K Jackson, G Findlay
}

\begin{abstract}
Aim-To investigate the usefulness of assaying endotoxin in non-directed bronchial lavage fluid (NBL), bronchoscopic bronchoalveolar lavage fluid (BAL), and sera as a means of diagnosing Gram negative, ventilator associated pneumonia. Methods-Samples from 64 patients were investigated. Fifty nine BALs and 92 NBLs were assayed in total including specimens taken during 28 episodes of clinical ventilator associated pneumonia (VAP).

Results-The concentration of endotoxin in BAL from patients with VAP developing within four days of commencing ventilation was significantly higher than in those without VAP $(p=0.015)$. There was no significant difference in endotoxin concentration in NBL or serum when comparing patients with and without VAP. A BAL endotoxin concentration of $6 \mathrm{EU} / \mathrm{ml}$ yielded the optimal operating characteristics (sensitivity, 81\%; specificity, 87\%; positive predictive value, $67 \%$; negative predictive value, 95\%). However, Gram stain of BAL provided the same information as quickly as the endotoxin assay and is considerably cheaper.

Conclusions-Despite its accuracy and rapidity, the BAL endotoxin assay must be shown to alter clinical management and patient outcome to be cost effective. (F Clin Pathol 2001;54:107-110)
\end{abstract}

Department of Medical Microbiology,

Llandough Hospital, Penlan Road, Penarth, Vale of Glamorgan, CF64 2XX, UK

P G Flanagan

Department of Medical Microbiology, University of Wales College of Medicine, Heath Park, Cardiff

CF4 4XN, UK

S K Jackson

Intensive Care Unit, University Hospital of Wales, Cardiff

CF4 4XW, UK

G Findlay

Correspondence to: Dr Flanagan paul.flanagan@phls.wales. nhs.uk

Accepted for publication 13 June 2000
Keywords: ventilator associated pneumonia; bronchoalveolar lavage; non-bronchoscopic lavage; endotoxin

Ventilator associated pneumonia (VAP) is the most common infection on intensive care units (ICU) with a high attributable mortality. ${ }^{1}$ The rapid diagnosis of VAP, allowing the same day selection of targeted antimicrobials, is an important clinical objective. Rapid diagnostic methods for VAP comprise microscopic identification of the aetiological agent by Gram or Giemsa staining of centrifuged deposits of respiratory tract specimens. In the UK, this usually involves centrifugation and microscopy with a $\times 100$ objective after Gram staining, but this has highly variable accuracy. ${ }^{2}$ Cytocentrifugation and Giemsa staining of bronchoscopic bronchoalveolar lavages (BALs) - to detect the proportion of intracellular bacteria within alveolar neutrophils and macrophageshas been advocated as a rapid, sensitive, and specific diagnostic technique. ${ }^{2}$ However, there is no agreement on the diagnostic threshold of intracellular bacteria and the accuracy may be reduced if antibiotics are introduced before respiratory sampling. ${ }^{2}$ Approximately $70 \%$ of cases of VAP are caused by Gram negative bacilli. ${ }^{13}$ Endotoxin (lipopolysaccharide; LPS) is a constituent of the outer membrane of all Gram negative bacteria and is shed into the local milieu during cell replication and death. ${ }^{4}$ Three studies have evaluated the diagnostic usefulness of measuring endotoxin in BAL fluid as a rapid diagnostic technique for Gram negative pneumonia ( $M$ Nys, et al, presented at the Immune Consequences of Sepsis Conference, Munich, Germany, March 1997). ${ }^{45}$ All three studies concluded that it was an accurate, clinically useful diagnostic tool in ventilated patients, although in one study only half of the patients were ventilated at the time. ${ }^{5}$ Nonbronchoscopic, non-directed bronchial lavage (NBL) is cheaper and more readily available than fibreoptic bronchoscopic (FOB) techniques. ${ }^{6}$ The accuracy of NBL is comparable with FOB methods. ${ }^{6}$ We investigated the diagnostic usefulness of assaying endotoxin in NBLs, BALs, and serum in the rapid diagnosis of Gram negative VAP.

\section{Methods}

The study population consisted of mechanically ventilated adult patients in three intensive care units, receiving mixed medical and surgical patients. Ethical approval was granted by the local ethics committee and informed written consent was obtained from relatives of the patients.

Patients were recruited at 72-96 hours of ventilation, at which time point, they underwent NBL and BAL, irrespective of whether they had clinical signs of pneumonia. Venous blood $(10 \mathrm{ml})$ was obtained aseptically at the same time. NBL and BAL were repeated if patients subsequently developed clinical signs of VAP.

Diagnostic criteria for VAP were modified from those of the CDC criteria for nosocomial pneumonia. ${ }^{7}$ Confirmed VAP was diagnosed by histopathology (antemortem or by necropsy performed within 96 hours of death) or as a result of concurrent isolation of a VAP pathogen from distal respiratory samples and from pleural fluid or blood culture, with no other apparent source of infection. A clinical diagnosis of VAP was made when chest $x$ rays showed (1) new, persistent (> 24 hours) and/or progressive infiltration, abscess, cavitation, or suspected empyema; (2) an increase in the volume and purulence of suctioned secretions; 
and (3) one or more of the following: fever ( $\geqslant 38^{\circ} \mathrm{C}$ for more than four hours), blood leucocytosis ( $\geqslant 11 \times 10^{9} /$ litre), and an increase in the fraction of inspired oxygen $\left(\mathrm{FiO}_{2}\right)$ of 0.2 needed to maintain arterial oxygen saturation, sustained for more than four hours. Patients remaining free of clinical signs of VAP had routine NBLs (not BAL) performed twice weekly thereafter.

\section{SAMPLING TECHNIQUES}

Specimens were taken sequentially within five minutes of each other, according to a random numbers design. The NBL technique was similar to that described previously. ${ }^{6}$ Briefly, a tracheal catheter (Argyle Sherwood Medical Ltd, London, UK) was attached to a sterile syringe filled with $20 \mathrm{ml}$ saline. The catheter was advanced down the endotracheal tube until resistance was met (at $\sim 50 \mathrm{~cm}$ ). The saline was injected over approximately 10 seconds and immediately reaspirated into the syringe. The volume of returned fluid was between 1 and $10 \mathrm{ml}$ (mean, 4). BAL was performed through a fibreoptic bronchoscope by experienced ICU physicians. ${ }^{3}$ After wedging of the instrument, $20 \mathrm{ml}$ of sterile saline was instilled and immediately reaspirated into a sterile syringe. The average volume of returned BAL fluid was $3.5 \mathrm{ml}$.

MICROBIOLOGICAL PROCESSING

Specimens were transported to the laboratory within 45 minutes of sampling, inoculated on to standard bacteriological media, and incubated at $37^{\circ} \mathrm{C}$. Gram stained smears of the NBL and BAL deposits were examined at high magnification $(\times 100$ objective $)$ for leucocytes and microorganisms. All plates were examined at 24 and 48 hours.

ENDOTOXIN ASSAYS

Aliquots of lavage specimens were collected in pyrogen free tubes before bacteriological processing and stored frozen at $-70^{\circ} \mathrm{C}$. Lavage fluids and standards with known concentrations of Escherichia coli 055:B5 endotoxin were thawed, centrifuged at $1800 \times g$ for 10 minutes and diluted $1 / 10$ in sterile water. All samples were batch tested in duplicate for LPS content by the kinetic chromogenic LAL assay (kinetic KQCL LAL assay; BioWhittaker UK Ltd, Wokingham, UK). A standard curve was constructed from the reaction times of the standard solutions and used to calculate the endotoxin concentration in the lavage fluids. To check for any inhibition/enhancement from the lavage, an aliquot of each sample was "spiked" with a known amount of endotoxin (0.5 EU). The results were rejected if they returned an LPS value $<50 \%$ or $>150 \%$ of $0.5 \mathrm{EU}$. Serum specimens were heated to $70^{\circ} \mathrm{C}$ for 10 minutes to eliminate false positives caused by serine proteases and then assayed identically to the lavages.

STATISTICAL ANALYSIS

The sensitivity, specificity, and predictive values were calculated using standard formulae. ${ }^{8}$ Analysis of the endotoxin data was performed using the Minitab version 10 statistical package. Differences in endotoxin content of samples were calculated by two sample $t$ tests.

\section{Results}

Sixty four patients were recruited (mean age, 54 years), comprising 38 general/cardiac surgical and 26 medical cases. Endotoxin was assayed in a total of 151 lavage fluids (92 NBLs and 59 BALs) obtained during a total of 97 sampling observations from 64 patients. In 77 of 97 observations, the patient had received antibiotics for at least 24 hours. Twenty eight of 64 patients developed clinical signs of VAP. Seven of 28 VAP episodes were confirmed by lung histopathology, concurrent bacteraemia, or coexistent pleural empyema with identical organisms in the pleural and lavage fluids. In 18 of 28 VAP episodes, both BAL and NBL endotoxin were assayed concurrently. In five episodes, BAL endotoxin alone was assayed. In another five episodes, NBL endotoxin alone was assayed because of an inadequate volume of NBL for the LPS assay or the unavailability of bronchoscopes. Five BALs and six NBLs yielded polymicrobial cultures.

\section{BAL ENDOTOXIN}

A total of 59 BALs were assayed for endotoxin (LPS), including 23 of 28 episodes of VAP. Of these 23 BAL samples, 16 yielded a Gram negative pathogen, either as the sole isolate (12 of 16) or as the predominant organism (four of 16). Five BALs yielded a Gram positive pathogen (four of five as the sole isolate) and two cultures were sterile. There was a significant difference in the mean concentration of BAL LPS between episodes of VAP $(161 \mathrm{EU} / \mathrm{ml})$ and observations of non-VAP $(20 \mathrm{EU} / \mathrm{ml}$; $\mathrm{p}=0.0002)$ when all episodes of VAP were analysed. There was also a significant difference in BAL LPS when the cohort of BALs taken within the first four days of ventilation was analysed $(275 \mathrm{EU} / \mathrm{ml}$ v $20 \mathrm{EU} / \mathrm{ml}$; $\mathrm{p}=0.015)$. The mean BAL LPS from Gram negative VAP was significantly higher than that from Gram positive VAP (239 EU/ml $v 14.6$ EU/MI; $p=0.0012)$. The optimal BAL LPS threshold occurred at $6 \mathrm{EU} / \mathrm{ml}$, with a sensitivity of $81 \%$ (Gram negative VAP only), specificity of $87 \%$ (calculated from total number of BALs from Gram positive VAPs $(n=5)$ and all non-VAP observations $(\mathrm{n}=36)$ ), positive predictive value (PPV) of $67 \%$, and negative predictive value (NPV) of $95 \%$. Figure 1 depicts the range of BAL endotoxin concentrations in VAP and non-VAP. Four BALs yielded normal oropharyngeal flora and LPS values of $0.05,0.6,34$, and $109 \mathrm{EU} / \mathrm{ml}$. One BAL from a patient without VAP had an LPS concentration of $500 \mathrm{EU} / \mathrm{ml}$ (fig 1) and yielded Gram negative bacteria at a bacterial load of $10^{6}$ $\mathrm{CFU} / \mathrm{ml}$.

NBL ENDOTOXIN

Ninety two NBLs were assayed in total. Twenty three of 92 were taken during episodes of VAP. Fifteen of 23 NBLs from these episodes yielded a Gram negative pathogen, either as the sole 
isolate (11 of 15$)$ or as the predominant organism (four of 15). Nine of 15 episodes occurred within four days of commencing ventilation. Five NBLs from VAP episodes yielded a Gram positive organism as the sole isolate (three of five) or the predominant pathogen and three yielded sterile cultures. There was no significant difference between mean NBL LPS from episodes of VAP $(114 \mathrm{EU} / \mathrm{ml})$ compared with observations of non-VAP (67 EU/ml; $\mathrm{p}=0.3$; fig 2). When analysing the cohort of NBLs taken within the first four days of ventilation, the difference in LPS between VAP and

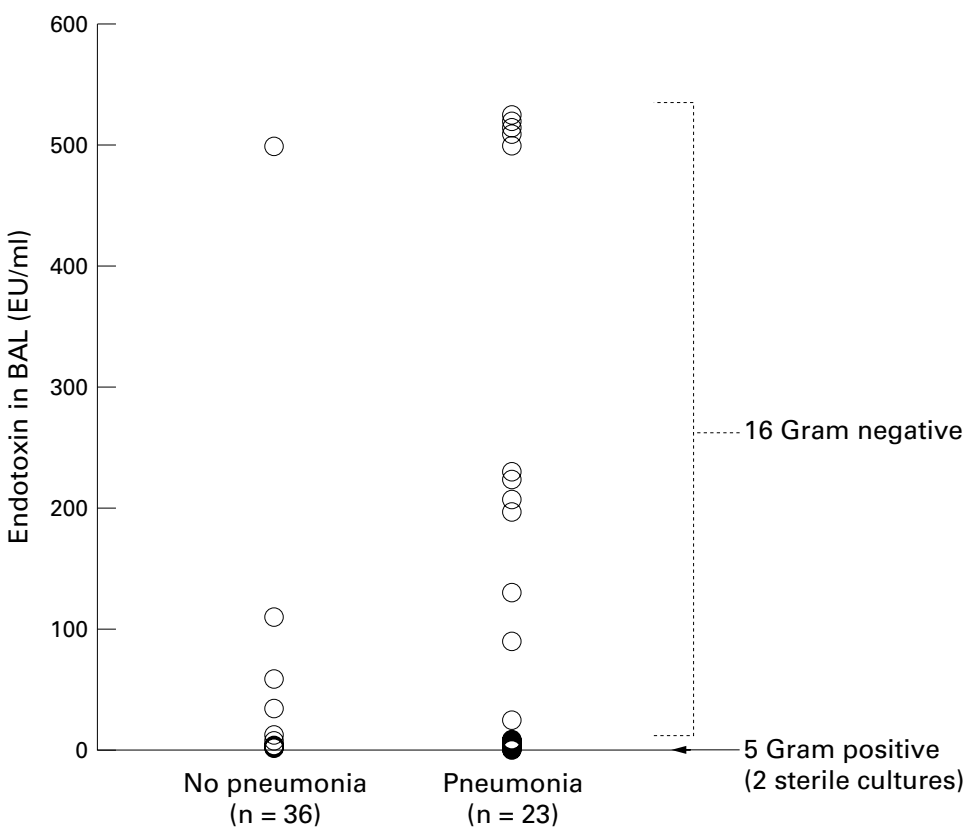

Figure 1 Endotoxin concentrations in bronchoscopic bronchoalveolar lavage fluid (BAL) from patients with and without clinical ventilator associated pneumonia (VAP). The Gram reaction of cultured organisms is shown beside the data points for the VAP episodes; $n$, number of BALs obtained from each group.

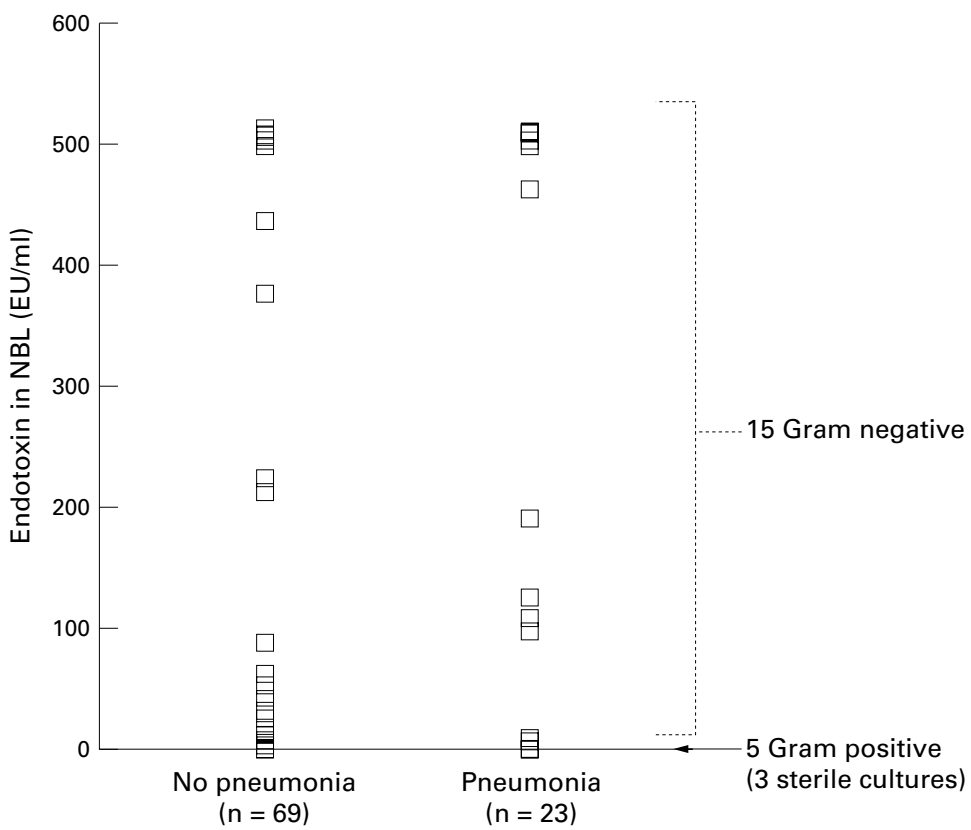

Figure 2 Endotoxin concentrations in non-directed bronchial lavage fluid samples (NBLs) from patients with and without clinical ventilator associated pneumonia (VAP). The Gram reaction of cultured organisms is shown beside the data points for the VAP episodes; $n$, number of NBLs obtained from each group.
non-VAP episodes still did not reach significance $(p=0.24)$. There was a significant difference between NBL LPS from Gram negative VAP compared with Gram positive VAP (mean, $179 \mathrm{EU} / \mathrm{ml} v 0.39 \mathrm{EU} / \mathrm{ml}$; $\mathrm{p}=0.00038)$. Three NBLs yielded normal oropharyngeal flora and LPS values of 53, 109, and $439 \mathrm{EU} / \mathrm{ml}$ (all non-VAP observations).

Thirty three sera (10 from episodes of VAP) were analysed for LPS content. All yielded LPS concentrations $<0.1 \mathrm{EU} / \mathrm{ml}$.

Eleven of 16 BAL Gram stains from episodes of Gram negative VAP were concordant with culture. The specificity, PPV, and NPV of the BAL Gram stain for Gram negative VAP were $88 \%$ (three of 25 BALs from non-VAP observations demonstrated Gram negative bacilli on Gram stain but not on subsequent culture), $73 \%$, and $89 \%$, respectively. Nine of 16 NBL Gram stains (56\%) from episodes of Gram negative VAP were concordant with culture. The specificity, PPV, and NPV of the NBL Gram stain for Gram negative VAP were $93 \%$ (two of 29 NBL Gram stains from non-VAP demonstrated Gram negative bacilli, not obtained from subsequent culture), $82 \%$, and $79 \%$, respectively.

\section{Discussion}

Our study indicates that assaying endotoxin in BAL within four days of commencing ventilation is an accurate, rapid diagnostic method for VAP. A BAL endotoxin value of $6 \mathrm{EU} / \mathrm{ml}$ or higher was predictive of VAP and had a comparable predictive value to Gram stain of BAL. There was no significant difference in endotoxin concentration in NBLs between VAP episodes and non-VAP observations $(p=0.3)$. The incidence of bronchial colonisation with nosocomial pathogens increases with the duration of ventilation. ${ }^{6}$ NBLs obtained after four days showed increasing concentrations of endotoxin in the absence of clinical VAP, reflecting lower respiratory tract colonisation with Gram negative bacteria and impaired specificity. This may also be true for BAL endotoxin assays, although our study design did not allow us to confirm this, because BALs were not repeated after recruitment in patients who remained free of VAP. Serum endotoxin values could not reliably identify patients with Gram negative VAP. This is consistent with previous studies of endotoxaemia ${ }^{9}$ and the pulmonary localisation of infection seen in most cases of VAP.

One of the purported advantages of BAL endotoxin assays is their diagnostic usefulness in patients who are started on appropriate antibiotics while awaiting bronchoscopy (M Nys, et $a l$, presented at the Immune Consequences of Sepsis Conference, Munich, Germany, March 1997). ${ }^{5}$ We found that most patients who received antibiotics and yielded sterile cultures had negligible lavage endotoxin values. This implies either that the patients did not have VAP and were thus given antibiotics unnecessarily or that endotoxin is rapidly cleared from lung secretions after antibiotic induced microbial death. 
The diagnostic accuracy of Gram stain of BAL was comparable to the endotoxin assay in contrast to previous studies, which found the LPS assay to be more accurate (M Nys, et al, presented at the Immune Consequences of Sepsis Conference, Munich, Germany, March 1997). ${ }^{4}$ To be cost effective, endotoxin assays must influence clinical management within hours of the clinical diagnosis of VAP, primarily by modifying antibiotic administration. If the endotoxin value was $<5 \mathrm{EU} / \mathrm{ml}$, antibiotics could be tailored to treat a possible Gram positive VAP or stopped and further clinical evaluation for a non-infectious cause could be performed. An endotoxin value above the threshold in a patient with clinical signs of VAP indicates Gram negative VAP in most cases. Previous studies report a high proportion of polymicrobial VAP $(\sim 40 \%),{ }^{1}$ and antibiotics with activity against Gram positive pathogens are still required until culture results are available. Thus, the scope for reducing antibiotic usage is probably limited.

The range of previously proposed thresholds of endotoxin ( $M$ Nys, et al, presented at the Immune Consequences of Sepsis Conference, Munich, Germany, March 1997) ${ }^{4}$ (5-10 EU/ $\mathrm{ml}$ ) reflects different patient populations and duration of ventilation. As such, a single fixed threshold is probably inappropriate and results within or close to this range should be regarded as equivocal and the patient managed according to the clinical evaluation.

Batch analysis of BAL/NBL specimens would not be practical in ICU and the cost for assaying a single specimen is approximately
$£ 20.00$. The BAL endotoxin assay has not yet been shown to be cost effective in terms of antibiotic usage. Whether it will influence patient outcome is uncertain. The diagnostic usefulness of assaying NBL and serum endotoxin is unconfirmed.

The authors are grateful to Dr JT Magee and Dr RA Barnes for reviewing the manuscript, Mrs J Parton for her valuable assistance in performing the endotoxin assays, and Drs D James and $S$ Leadbeatter for evaluating the lung histopathology. Supported by grants from the Welsh Scheme for the Development of Health and Social Research, Hoescht Marion Roussell Inc, and Pfizer Limited.

1 Bowton DL. Nosocomial pneumonia in the ICU-year 2000 and beyond. Chest 1999;115:28S-33S.

2 Baselski VS, el-Torky M, Coalson JJ, et al. The standardisation of criteria for processing and interpreting laboratory specimens in patients with suspected ventilator-associated pneumonia. Chest 1992;102:57IS-9S.

3 de Jaeger A, Litalien C, Lacroix J, et al. Protected specimen brush or bronchoalveolar lavage to diagnose bacterial nosocomial pneumonia in ventilated adults: a metaanalysis. Crit Care Med 1999;27:2548-60.

4 Pugin J, Auckenthaler R, Delaspre 0, et al. Rapid diagnosis of Gram-negative pneumonia by assay of endotoxin in bronchoalveolar lavage fluid. Thorax 1992;47:547-9.

5 Kollef MH, Eisenberg PR, Ohlendorf MF, et al. The accuracy of elevated concentrations of endotoxin in bronchoalveolar lavage fluid for the rapid diagnosis of Gram-negative pneumonia. Am $\mathcal{f}$ Respir Crit Care Med 1996;154:1020-8.

6 A'Court CHD, Garrard CS, Crook D, et al. Microbiological lung surveillance in mechanically ventilated patient using non-directed bronchial lavage and quantitative culture. $Q \mathcal{F}$ Med 1993;86:635-48.

7 Centers for Disease Control. CDC definitions for nosocomial infections. Am Rev Respir Dis 1988;139:1058-9.

8 Swinscow T. Statistics at square one, 8th ed. Plymouth: Latimer Trend and Company, 1983.

9 Elin RJ, Hosseini J. Clinical utility of the limulus amoebocyte lysate (LAL) test. Prog Clin Biol Res 1985;189: 307-27. 